\title{
Incidencia y Evitabilidad de Eventos Adversos en Pacientes Hospitalizados en tres Instituciones Hospitalarias en Colombia, 2006
}

\author{
Incidence and preventability of adverse events in \\ patients hospitalised in three Colombian hospitals \\ during 2006
}

Hernando Gaitán-Duarte ${ }^{1}$, Javier Eslava-Schmalbach ${ }^{1}$, Nelcy Rodríguez-Malagon ${ }^{1}$, Víctor Forero-Supelano², Dagoberto Santofimio-Sierra ${ }^{3}$, Hernando Altahona ${ }^{4}$, y Grupo de Evaluación de Tecnologías y Políticas en Salud

1 Instituto de Investigaciones Clínicas, Facultad de Medicina, Universidad Nacional de Colombia. hggaitand@unal.edu.co

2 Clínica Juan N. Corpas Ltda. Bogotá, Colombia

3 Hospital Universitario Hernando Moncaleano Perdomo de Neiva. Colombia

4 Fundación Universitaria Sanitas. Bogotá, Colombia

Recibido 8 Noviembre 2007/Enviado para Modificación 22 Marzo 2008/Aceptado 11 Abril 2008

\section{RESUMEN}

Objetivo Determinar incidencia, clasificación, evitabilidad e impacto de los Eventos Adversos (EA) para establecer su importancia como problema en algunos hospitales en Colombia.

Metodología Cohorte prospectiva de pacientes hospitalizados al menos 12 horas en 3 instituciones generales en Colombia. Se excluyeron pacientes psiquiátricos, presencia del EA antes del ingreso a la hospitalización índice. Con una diferencia estimada del $1 \%$ (complejidad alta y media), significancia: 0,05\%, poder: $80 \%$ relación 2:1 se requerían mínimo 6557 pacientes. Fuente de información: historia clínica Fase I: traducción y adaptación de formularios. Fase II: vigilancia activa de eventos de tamización. Fase III: evaluación de la asociación del EA con el cuidado proveído, por un comité de especialistas, Variables: edad, género y tipo de afiliación al sistema de seguridad social, incidencia acumulada de EA, temporalidad, evitabilidad y discapacidad resultante naturaleza del EA.

Resultados Fueron evaluados 6688 sujetos durante el periodo de vigilancia. Se detectaron 505 pacientes con eventos de tamización positivos (7,9 \% IC95 \% 7,3$8,6)$. Un total de 310 sujetos presentaron al menos un evento adverso; Incidencia acumulada: (4,6 \% IC95 \% 4,1-5,1) durante la hospitalización. De estos se consideró evitable el evento adverso en 189 sujetos (61 \%, IC95 \% 55-66). Ocurrió discapacidad permanente en $1,3 \%$. La mortalidad asociada al evento adverso fue de $6,4 \%$ (20/310). Se incrementó la hospitalización como consecuencia del EA un total de 1072 días. 
Conclusiones La incidencia de eventos adversos evitables fue relevante en los tres hospitales de Colombia.

Palabras Clave: Administración de la seguridad, errores médicos, servicios de salud, hospitalización (fuente: DeCS, BIREME).

\section{ABSTRACT}

Objective Determining adverse event (AE) incidence, preventability, classification and impact for establishing their importance as a public health problem within the Colombian Social Security System.

Methodology This was a study of a prospective inpatient cohort from three Colombian general-practice institutions. Inclusion criteria: at least 12 hours' length of hospital stay during 2006. Exclusion criteria: suffering psychiatric disorders and AE which had occurred before hospitalisation indexing. The sample consisted of 6557 patients. Source of information: clinical charts. Being a three-phase design, the first phase consisted of translating and standardising screening and causation formats, phase II of actively monitoring screening criteria and phase III of evaluating causation regarding the care being provided, based on specialist committee concept on a 0-6 scale. The variables measured were age, gender, social security affiliation, cumulative $A E$ incidence, temporality, preventability of $A E$ and disability resulting from them.

Results 6688 patients were evaluated; 505 of them fulfilled positive screening criteria (95\% $\mathrm{Cl}=7,9 ; 7,3-8,6), 310$ presented at least one $\mathrm{AE}$ during their hospitalisation (95 $\% \mathrm{Cl}$ for accumulated incidence $=4,6 ; 4,1-5,1)$. AE were considered to have been preventable in 189 cases (95\% $\mathrm{Cl}=61 ; 55-66)$ and permanent disability occurred in 1,3 $\%$ of them. AE-associated mortality was $6,4 \%$ (20/310). Hospitalisation became increased to 1072 days as a direct consequence of AE.

Conclusions This study revealed an important incidence of $A E$ in three Colombian hospitals, these being mainly preventable. Their ongoing monitoring as a part of risk management systems could reduce costs and AE-associated morbidity and mortality.

Key Words: Safety management, medical error, health service, adverse effect, day care (source: $M e S H, N L M$ ).

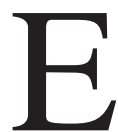

n los últimos años ha crecido el interés de las organizaciones sanitarias por mejorar la seguridad en la provisión de los cuidados de salud en quienes son atendidos en sus instituciones. Lo relativo a eventos adversos, también denominados por algunos autores como eventos reportables (1) cobra importancia en el tema de gestión de la calidad en salud. Un evento adverso es una situación que termina en daño no intencional al paciente, que ocurre por causa o con ocasión del servicio y es descubierta después de que éste ingresa a la institución (1). Se detectan a través de los eventos centinelas o de tamización definidos como eventos no deseados que señalan que algo serio ha ocurrido y que requieren una investigación más profunda (1). Es un indicador de la seguridad en la atención (2). 
La frecuencia de los eventos adversos (EA) varía entre 1 y 22 \% dependiendo del servicio donde son medidos (2-7) del tipo de institución (2, 8,9), y del método utilizado para su medición $(10,11,12)$. Se ha reportado que un 30 a $70 \%$ de ellos son evitables $(5,13)$ y tienen un significativo impacto en términos de prolongación de la estancia hospitalaria, discapacidad resultante, costos y mortalidad $(14,15)$.

Los EA han sido clasificados según la causa básica u origen del evento (2), la seriedad, la discapacidad resultante (16), la severidad (6), el componente de la atención donde se origina y la temporalidad de la aparición del evento con respecto al ingreso en la hospitalización índice (2).

Poco se conoce en países en desarrollo y en el contexto local sobre la frecuencia de los EA en hospitales generales, así como los aspectos relacionados a su evitabilidad y discapacidad resultante. El objetivo de este trabajo fue determinar la incidencia, evitabilidad, clasificación e impacto de los EA en el ambiente hospitalario en Colombia con el propósito de determinar su posible importancia como problema de salud pública en el nivel local.

\section{MATERIALES Y MÉTODOS}

Cohorte concurrente de pacientes hospitalizados al menos 12 horas entre el 15 de mayo y 15 de octubre de 2006, pertenecientes a: una institución pública de referencia de alta complejidad, tipo "hospital universitario" ubicado la región sur occidental del país; una institución privada de complejidad media que atiende preferentemente pacientes del Régimen Subsidiado; y una institución privada que atiende preferentemente pacientes del Régimen Contributivo y atención particular. Estas dos últimas estaban ubicadas en la ciudad de Bogotá.

Dichas instituciones tienen más de 100 camas y atienden más de 4000 hospitalizaciones por año, 24 horas al día. Fueron seleccionadas Por su disposición gerencial de participar en el estudio. Se incluyeron pacientes de los servicios de obstetricia, quirúrgicos y médicos. Se excluyeron pacientes psiquiátricos y trasladados de otras instituciones por complicaciones o con eventos adversos originados antes de la hospitalización inicial. El protocolo de investigación fue aprobado en los Comités de Ética e Investigaciones en cada una de las instituciones de salud participantes y se firmó un acuerdo de confidencialidad de la información y los resultados para cada entidad. 
En el programa EpiInfo ${ }^{\circledR}$ se estimó una muestra mínima de 6557 pacientes con una frecuencia estimada de EA del 2,2 \% para instituciones de alta complejidad y $1,2 \%$ en nivel medio de complejidad (error alfa $=0,05$; poder $=80$ $\%$, relación 2:1). El estudio fue diseñado en tres fases. En la primera fase se desarrolló la estandarización en español de los instrumentos sugeridos por Baker y cols (2). Dos investigadores tradujeron el texto del inglés al español. El documento resultante fue sometido a validación de apariencia y contenido por un comité de expertos. Se realizó una prueba piloto con cada uno de los encuestadores. Al finalizar esta fase se obtuvieron 3 instrumentos para el tamizaje, la evaluación de la asociación, la evitabilidad y la discapacidad, respectivamente.

En la fase dos se capacitaron enfermeras profesionales para la vigilancia activa de pacientes hospitalizados. Este procedimiento fue llevado diariamente durante la hospitalización índice o inicial por medio del registro de los criterios de tamización (Tabla 1), variables sociodemográficas y la comorbilidad por medio del índice de Charlson (17). Se tomaron en cuenta los eventos adversos que se originaron en la hospitalización índice durante el periodo de estudio y hasta el día 30 del alta. En caso de presentarse dos o tres criterios de tamización relacionados entre si se seleccionó un solo evento, el que primero se presentó y originó los eventos subsecuentes. En la situación de presentarse dos o más eventos centinelas con poca posibilidad de asociación entre ellos, se tomaron en cuenta todos los eventos. Fueron registradas las siguientes variables: edad, género y tipo de afiliación al sistema de seguridad social (seguro privado, seguro social contributivo, seguro social subsidiado por el estado y ausencia de seguridad social). Para evaluar la concordancia interobservador, se tomó una muestra aleatoria de 60 formularios por institución que mostró un índice de Kappa de 0,67 como máximo, que muestra una reproducibilidad considerada como moderada (18).

En la fase tres, los casos con al menos un criterio de tamización fueron llevados a comités de evaluación, constituidos por grupos de al menos dos especialistas del área pertinente. Se calificó como EA aquellos eventos de tamización en los que el comité asigno un puntaje mayor o igual a tres en la escala de asociación de uno a seis. A partir de este puntaje se estableció si el daño ocurrido estaba asociado con el cuidado provisto (2). Adicionalmente, estos comités calificaron la seriedad, la temporalidad, el tipo de evento, la deficiencia asociada y la evitabilidad (Cuadro 1). 
Dado que solamente se consideraron eventos ocurridos durante la hospitalización índice la temporalidad se clasificó como detectada en la misma hospitalización, durante el control ambulatorio, o en la rehospitalización. El tipo de deficiencia que origino el evento se clasificó como: deficiencia en el desempeño, en la prevención, en el diagnostico, en el tratamiento con medicamentos y deficiencias de los sistemas. En cuanto a la evitabilidad del evento, se consideró como evento adverso evitable aquel evento que no hubiese ocurrido si se hubieran seguido los estándares de cuidado rutinarios y cotidianos apropiados para el momento del estudio (19). Se clasificó la estancia prolongada como el número de días de hospitalización mayor al estándar para el manejo de una patología, según concepto del panel de expertos. La mortalidad asociada fue el número de muertes asociada a eventos reportables. En casos de desacuerdo se recurrió al concepto de un tercer evaluador. La discapacidad se definió como daño en la función física o mental. Se categorizó como: recuperación en un mes, recuperación de uno a seis meses, recuperación en un periodo mayor a seis meses, daño permanente con menos de $50 \%$ de discapacidad, daño permanente con más de un $50 \%$ de discapacidad y muerte. Fue evaluada por un experto en salud ocupacional quien estuvo enmascarado al lugar de origen de la información. Se utilizó el manual de calificación de invalidez del Ministerio de la Protección Social de Colombia (20)

Cuadro 1. Formularios de calificación del Eventos Adversos

¿El evento está asociado con el cuidado proveído o con ocasión del mismo?

1. Definitivamente el evento se debió a la enfermedad de base

2. El evento muy probablemente se debió a la enfermedad de base.

3. Probablemente el evento se debió a la enfermedad, pero podría haber estado relacionado con la provisión del servicio o con ocasión de este.

4. Probablemente el evento se debió más a la provisión del servicio o con ocasión de este, que a la enfermedad de base.

5. El evento muy probablemente se debió a la provisión del servicio o con ocasión de este.

6. Casi con seguridad el evento se debió a la provisión del servicio o con ocasión de este.

¿Qué nivel de evitabilidad tenía el evento reportado?

1. Definitivamente el evento no pudo haber sido evitado.

2. Muy probablemente el evento no pudo haber sido

evitado.

3. Probablemente el evento no pudo haber sido evitado.

4. Probablemente el evento pudo haber sido evitado.

5. Muy probablemente el evento pudo haber sido evitado.

6. Definitivamente el evento pudo haber sido evitado. 
La fuente de información fue la historia clínica: notas de ingreso notas de evolución, notas de enfermería, registros de anestesia, etc. Se realizó verificación de la calidad de la información.

Análisis estadístico: Se describe la frecuencia de los tipos de eventos tamizados. La frecuencia de eventos centinelas positivos se estimó como: número de sujetos con al menos un evento centinela/ número de sujetos a riesgo. La incidencia acumulada de sujetos con eventos adversos se estimó como número de pacientes con al menos un evento adverso/número total de pacientes en riesgo. Adicionalmente se estimó la proporción de eventos reportables evitables, de eventos serios y de discapacidad resultante. Para el análisis estadístico de la información se utilizó STATA 8.0.

\section{RESULTADOS}

Durante el periodo a estudio se presentaron en las tres instituciones 7263 admisiones que correspondieron a un total de 6688 pacientes (Figura 1). Ingresaron a servicios quirúrgicos 2361 (35,3\%), a obstetricia 2732 (41 \%) y a servicios médicos 1595 (23,9\%). De los pacientes incluidos, 4839 correspondieron a mujeres (72,5\%) y 1849 correspondieron a hombres $(27,5 \%)$.

La edad en el grupo de pacientes con especialidades quirúrgicas tuvo una mediana de 49 años [16-86], en el grupo de especialidades médicas fue 63 años [16-91] y en ginecobstetricia 29 años [19-69]. El $44 \%$ tenían seguros privados de salud, 28,3\% seguridad social contributiva, el 21,5\% a la seguridad social subsidiada y el 5,4\% ningún tipo de aseguramiento. El 78 \% ingresaron como urgencia. El 30 \% de los pacientes tuvo una puntuación mayor o igual a 1 en el índice de comorbilidad de Charlson.

Se detectaron 789 eventos centinelas en 505 pacientes (frecuencia de eventos centinelas positivos: 7,9 \% IC95 \% 7,3-8,6) Tabla 1. La incidencia acumulada de EA fue de 4,6 \% (310 pacientes; IC95 \% 4,1-5,1). La incidencia de EA en especialidades quirúrgicas fue: 6,2 \% (IC95 \% 6,3-7,2), ginecobstetricia: (3,4 \% IC95 \% 2,8-4,2) y especialidades médicas: (3,6 \% IC95 \% 2,8-4,7). El número total de eventos adversos en todas las hospitalizaciones fue de 450; un $22 \%$ presentaron dos o más EA y un $4 \%$ presentaron tres o más eventos adversos. 
En cuanto a la temporalidad de los eventos en relación al momento de la hospitalización, 69 \% (218/310) se detectó durante la hospitalización, 35 \% (110/310) durante la rehospitalización y 7 \% (24/310) en cuidado ambulatorio. El sitio donde más frecuentemente se presentaron los EA fueron en salas de cirugía (31,0\%), en hospitalización (28,8 \%), en salas obstetricia (17,7 \%), en urgencias (9,4\%), en unidad de cuidados intensivos (6,7 \%) y en recuperación postquirúrgica (6,3\%). Los EA se presentaron más frecuentemente como complicaciones técnicas intraoperatorias (27,6\%), complicaciones no técnicas (15,7\%), relacionadas con procedimientos obstétricos (parto-posparto) de enfermería (16,4\%), infección de la herida (12,5\%), relacionados con medicamentos (9,2\%), complicaciones tardías (6,5\%), las fallas diagnosticas, las fallas terapéuticas y las caídas tuvieron una frecuencia relativa del $3 \%$ cada una. Sólo 1,5 \% se relacionaron con componentes sanguíneos y 0,6 \% con fallas técnicas. Las deficiencias que originaron la aparición del EA se observan en la Tabla 2.

Figura 1. Flujograma de pacientes

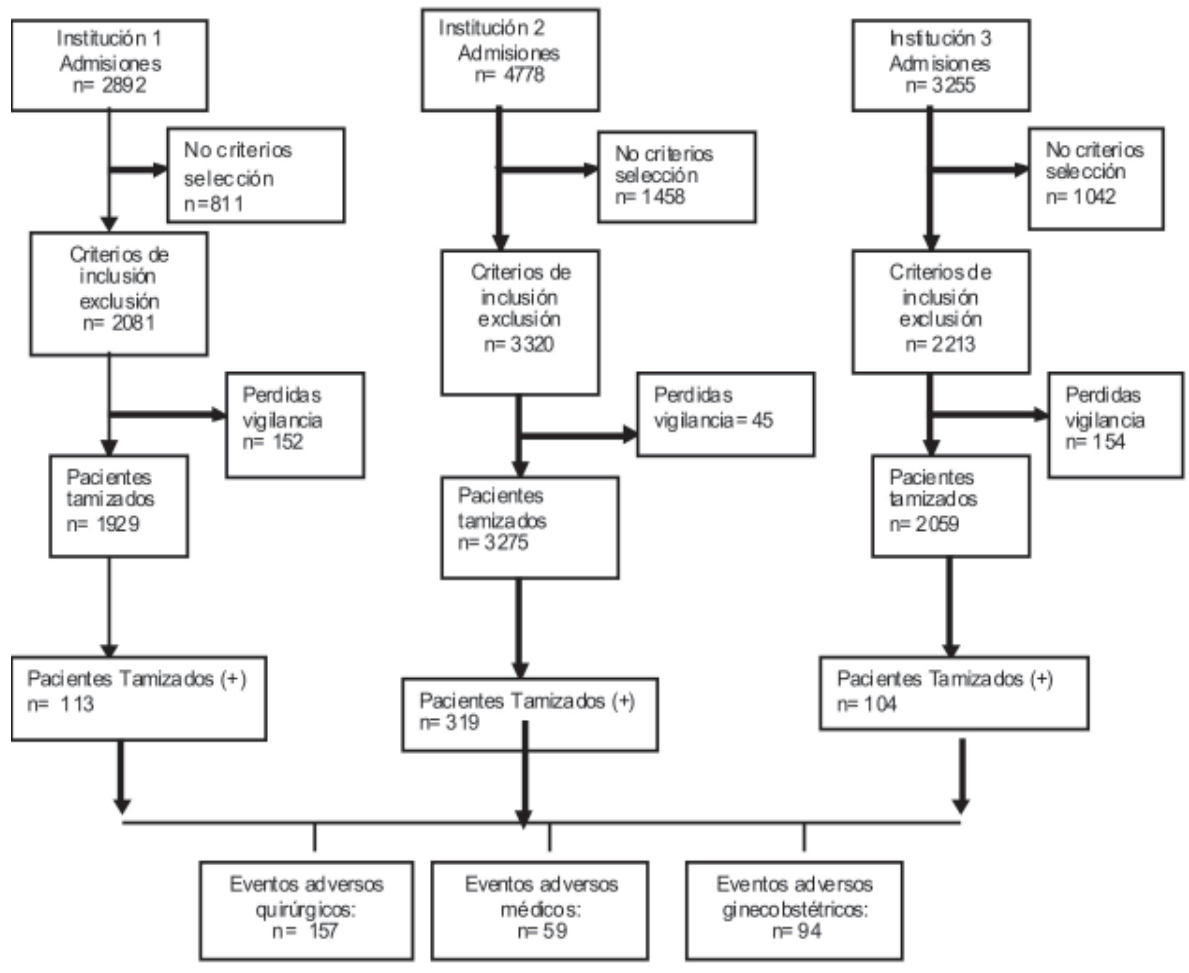


Se consideró que eran eventos probablemente evitables 189 de los 310 eventos (61 \%, IC95 \% 55-66). Al observar la evitabilidad por especialidad se encontró que en especialidades quirúrgicas fue: (58 \%, IC95 \% 49-65), en ginecobstetricia (70 \% IC95 \% 59-79), en especialidades medicas: (54 \% IC95 \% 40-67). De estos eventos, casi con seguridad era evitable el $30 \%$.

En 61 \% de los pacientes con EA, se aumentó la hospitalización como consecuencia de este con un incremento total de 1072 días. Esta adición tuvo una mediana de 2 días con un rango de 1 a 54 días. Se requirió alguna intervención quirúrgica adicional en el 39 \% de los pacientes con EA. El 72 \% de los EA fueron considerados como eventos serios. Del total de pacientes 251 tuvieron discapacidad menor a 6 meses (80 \%, IC95 \% 76-85) y 13 pacientes tuvieron una recuperación mayor a 6 meses (4 \%, IC 95 \% 2-7). Hubo daño permanente en 4 pacientes. La mortalidad asociada al evento adverso fue de $(6,4 \%)$.

Tabla 1. Frecuencia de eventos de tamización en tres instituciones hospitalarias en Colombia, 2006

\begin{tabular}{|c|c|}
\hline Evento de tamización & $\begin{array}{l}\text { Número } \\
\text { Tamizado }\end{array}$ \\
\hline $\begin{array}{l}\text { Readmisión no planeada como resultado del cuidado general dado al } \\
\text { paciente. }\end{array}$ & 221 \\
\hline Remisión no planeada a otro hospital de igual o mayor complejidad. & 13 \\
\hline $\begin{array}{l}\text { Remisión no planeada a la unidad de cuidado intensivo u otra área de } \\
\text { cuidado especial. }\end{array}$ & 105 \\
\hline Traslado no planeado o reingreso a sala de cirugía. & 79 \\
\hline Muerte inesperada. & 10 \\
\hline Paro cardiorrespiratorio inesperado. & 9 \\
\hline $\begin{array}{l}\text { Extracción, lesión o reparación de un órgano o estructura, no planeada } \\
\text { durante la cirugía. }\end{array}$ & 54 \\
\hline $\begin{array}{l}\text { Lesiones o complicaciones relacionadas con el trabajo de parto, el parto o el } \\
\text { aborto, incluyendo complicaciones neonatales. Puede incluir remoción, lesión } \\
\text { o reparación de un órgano o estructura }\end{array}$ & 50 \\
\hline $\begin{array}{l}\text { Complicaciones intrahospitalarias desarrolladas durante el proceso de } \\
\text { admisión. }\end{array}$ & 8 \\
\hline $\begin{array}{l}\text { Desarrollo de un déficit neurológico que no estaba presente en el momento } \\
\text { de la admisión (incluyendo cambio en el nivel de conciencia o delirio) }\end{array}$ & 9 \\
\hline Infección nosocomial o infección adquirida en el hospital. & 144 \\
\hline Accidente o lesión del paciente ocurridos en el hospital. & 17 \\
\hline $\begin{array}{l}\text { Reacción adversa a medicamento/ insumo indicado en la Historia clínica o en } \\
\text { el Reporte de reacciones adversas a medicamentos / insumos (incluido } \\
\text { hemoderivados). }\end{array}$ & \\
\hline Cualquier otro desenlace no cubierto por los anteriores criterios. & 34 \\
\hline Total & 789 \\
\hline
\end{tabular}




\section{DISCUSIÓN}

Este estudio es la primera aproximación epidemiológica publicada sobre el tema en Colombia y muestra que al menos el (4,5\%) de los pacientes hospitalizados en servicios quirúrgicos, médicos o de ginecobstetricia de las tres instituciones observadas presentaron eventos adversos. Estos resultados son ligeramente mayores a los informados por estudio de Brennan en Estados Unidos quien presentó una incidencia del (3,7 \%) (21) e inferior a las incidencias publicadas por Baker (2) del (7,5 \%) y Forster (9) en Canadá, Davis y cols del (11,3 $\%$ )en Nueva Zelandia (21) y Vincent con el (11,7 \%) en el Reino Unido (4).

Tabla 2. Deficiencia que originó el evento adverso en tres instituciones hospitalarias en Colombia, 2006

\begin{tabular}{lr}
\hline Deficiencia en el desempeño & $\%$ \\
\hline Error técnico & 57,5 \\
\hline Demora evitable en el tratamiento & 12 \\
Monitoria inadecuada después de un procedimiento & 15 \\
\hline Preparación inadecuada antes de un procedimiento (6 \%) & 6 \\
\hline Uso de una Terapia inadecuada u obsoleta & 3 \\
Otras causas & 6,5 \\
\hline Deficiencia en la prevención & 18 \\
Demora evitable en el tratamiento & 61 \\
\hline Falta para tomar precauciones para prevenir la lesión & 3,5 \\
\hline Falta para utilizar los exámenes indicados & 17 \\
\hline Otras causas & \\
Deficiencias en el diagnostico & 61 \\
\hline Demoras evitables en el proceso diagnostico & 35 \\
Falla para reaccionar frente a los hallazgos o resultados & 14 \\
\hline Falla para solicitar los exámenes indicados & \\
Deficiencias en el tratamiento con medicamentos & 45 \\
\hline Debidos a falla para reconocer interacción medicamentosa & 26 \\
Demora evitable para iniciar el tratamiento terapéutico & 13 \\
\hline Dosis en el método de uso & 6 \\
\hline Dosis inadecuadas o medicamentos contraindicadas & 11 \\
\hline Otras & \\
Deficiencias sistémicas & 25 \\
\hline Demora en la provisión del cuidado o plan de atención & 16 \\
\hline Problemas con los equipos o los suministros & 25 \\
\hline Inadecuado funcionamiento del servicio hospitalario & 21 \\
\hline Inadecuada comunicación & 13 \\
\hline Otros
\end{tabular}

Las diferencias en la incidencia se podrían explicar por las características de la población incorporada a este estudio, en el que predominó la población obstétrica, en la cual se ha informado una frecuencia de EA del 1,5 al 4,0 \% $(4,21,23)$. Así mismo, los reportes indican que el EA es más frecuente en pacientes mayores a 70 años (18). En nuestra población la mediana de edad 
en los tres grupos fue menor a esta edad. Este hecho puede ser relacionado con la baja mortalidad presente en nuestro estudio, la cual es menor a la reportada por Baker (2) y similar a la de Wilson (5).

Se presentó un subregistro derivado de perdidas en el seguimiento de pacientes no mayor al $10 \%$ y es posible un subregistro de EA del 20 al $46 \%$ dependiendo del método de detección y del servicio (10). Generalmente, los estudios respecto a este tema han utilizado solo la historia clínica como fuente de información para la detección de eventos adversos institucionales, teniendo limitaciones esta fuente de información (24). Por otra parte, podría haber influido el haber incluido sólo un evento de tamización, cuando estaban relacionados, así como el hecho de no haber tomado en cuenta los eventos adversos previos a la hospitalización.

Se determinó escoger un punto de corte inferior al utilizado en los estudios previos (puntaje igual o mayor 4 en una escala de 6) debido a una tendencia de los comités institucionales a subestimar la asociación con el cuidado provisto detectada por los médicos monitores. De esta manera se buscó incluir todos eventos que pudieron estar asociados al cuidado provisto. Este tema será revisado ampliamente en una publicación posterior, referente a los aspectos cualitativos de la medición de los eventos adversos.

En cuanto a la evitabilidad del $61 \%$, esta es una cifra ligeramente superior a la informada en el estudio de Davis con 58 \% (22), por Vincent con 48 \% y Baker (36,8 \%). Esto probablemente se debió a que en la población obstétrica predominan los eventos evitables $(4,10)$.

En conclusión, la información analizada da cuenta de una importante incidencia de los eventos adversos evitables en tres hospitales de Colombia. Su medición permanente podría favorecer la reducción de costos en salud, así como la morbilidad asociada a eventos adversos

Agradecimientos. Al Instituto Colombiano de Ciencia y Tecnología COLCIENCIAS que financió este proyecto mediante el Grant identificado con el código: 1101-04-18198 y la Universidad Nacional de Colombia, División de Investigación de la Sede Bogota DIB Código: 20201007427. Grupo de Evaluación de tecnologías y políticas en Salud: Por la Universidad Nacional de Colombia: Hernando Gaitán Duarte, Ingrid Arevalo, Javier Eslava Schmalbach, Nelcy Rodríguez Malagon, Ariel Ruiz Parra, Edith Ángel Muller, Oscar Guevara, Jacinto Sánchez A, Andrés Rubio, Carlos Pacheco Consuegra, Andrea Rodríguez, María Teresa Vallejo, patricia Ojeda Espis. Por la Fundación Universitaria Sanitas: Hernando Altahona, Nancy Yomayusa González, Carlos Camargo Mila, Álvaro Sarmiento Orjuela, Mauricio Herrera Méndez, José 
Francisco Londoño Borda. Por la Clínica Juan N Corpas: Víctor Hugo Forero, Bernardo Páez Fonseca, Hernán Roa Lemus, Gonzalo Bernal Ferreira, Federico Fernández Bernal, Ricardo Mendoza Ramírez, José Bohórquez Reyes, William Buitrago González, Álvaro Medina Ferro, Denny Luengas Suárez, Myriam Suárez Cortés. Por el Hospital Universitario de Neiva: Dagoberto Santofimio, Ángela María Salcedo, Abner Lozano, Álvaro Cerrato Girón, Fabio Rojas, Flavio Vargas, Luis Fernando Duran, Gustavo Poveda, Luis Arturo Rojas Charry.

\section{REFERENCIAS}

1. Reportable Events Guidelines. Ministry of Health New Zeland. Disponible en: http:// uww.moh.govt.nz/moh.nsf/O/DDFCEFCD693AEBC4CC256AD0007F41BB Consultado 1 de Abril de 2008.

2. Baker GR, Norton PG, Flintoft V, Blais R, Brown A, Cox J, et-al. The Canadian Adverse Events Study: the incidence of adverse events among hospital patients in Canada. CMAJ 2004; 170:1678- 86.

3. Woods D, Thomas E, Holl J, Altman S, Brennan T. Adverse events and preventable adverse events in children. Pediatrics. 2005; 115:155-60.

4. Vincent $\mathrm{Ch}$, Neale G, Woloshynowych. Adverse events in British hospitals: preliminary retrospective record review. BMJ, 2001,322: 517-519.

5. Wilson RM, Runciman WB, Gibberd RW, Harrison BT, Newby L, Hamilton JD. The Quality in Australian Health Care Study. Med J Aust. 1995; 163:458-7.

6. Rothschild JM, Landrigan CP, Cronin JW, Kaushal R, Lockley SW, Burdick E, et al. The Critical Care Safety Study: The incidence and nature of adverse events and serious medical errors in intensive care.Crit Care Med. 2005; 33:1694-700.

7. Kable AK, Gibberd RW, Spigelman AD. Adverse events in surgical patients in Australia. Int J Qual Health Care. 2002; 14:269-76.

8. Thomas EJ, Orav EJ, Brennan TA. Hospital ownership and preventable adverse events. J Gen Intern Med. 2000; 15:211-9.

9. Forster AJ, Asmis TR, Clark HD, Al Saied G, Code CC, Caughey SC, et al. Ottawa Hospital Patient Safety Study: incidence and timing of adverse events in patients admitted to a Canadian teaching hospital. CMAJ. 2004; 170:1235-40.

10. Michel P, Quenon JL, de Sarasqueta AM, Scemama O. Comparison of three methods for estimating rates of adverse events and rates of preventable adverse events in acute care hospitals. BMJ. 2004; 328:199- 203.

11. Allan EL, Barker KN. Fundamentals of medication error research. Am J Hosp Pharm. 1990; 47:555- 71.

12. Andrews LB, Stocking C, Krizek T, Gottlieb L, Krizek C, Vargish T, Siegler M. An alternative strategy for studying adverse events in medical care. Lancet.1997; 349:30913.

13. Sheldon T. Dutch study shows that $40 \%$ of adverse incidents in hospital are avoidable. BMJ. 2007;334:925.

14. Ehsani JP, Jackson T, Duckett SJ. The incidence and cost of adverse events in Victorian hospitals 2003-04.Med J Aust. 2006; 184:551-5.

15. Garcia-Martin M, Lardelli-Claret P, Bueno-Cavanillas A, Luna-del-Castillo JD, Espigares-Garcia M, Galvez-Vargas R. Proportion of hospital deaths associated with adverse events. J Clin Epidemiol. 1997; 50:1319-26.

16. Forster AJ, Murff HJ, Peterson JF, Gandhi TK, Bates DW. The incidence and severity of adverse events affecting patients after discharge from the hospital. Ann Intern Med. 2003; 138:161-7.

17. Schneeweiss S, Maclure M. Use of comorbidity scores for control of confounding in studies using administrative databases. Int J Epidemiol. 2000; 29:891-8. 
18. Landis RJ, Koch GG. The measurement of observer agreement for categorical Data. Biometrics 1977;33:159 -174.

19. Thomas EJ, Brennan TA. Incidence and types of preventable adverse events in elderly patients: population based review of medical records. BMJ 2000; 320:741-4.

20. Ministerio de trabajo y Seguridad Social, Colombia. [Internet] Decreto 917 del 28 de Mayo de 1999. Manual Único para la Calificación de la Invalidez. Disponible en http:// www.suratep.com/legislacion/articulos/192/index.php. Consultado: 16 de octubre de 2007.

21. Brennan TA, Leape LL, Laird NM, Hebert L, Localio AR, Lawthers AG, et al Incidence of adverse events and negligence in hospitalized patients. Results of the Harvard Medical Practice Study I. N Engl J Med. 1991; 324:370-6.

22. Davis $P$, Lay-Yee R, Briant $R$, Scott A. Preventable in-hospital medical injury under the "no fault" system in New Zealand. Qual Saf Health Care. 2003; 12:251-6.

23. Forster AJ, Fung I, Caughey S, Oppenheimer L, Beach C, Shojania KG, van Walraven C. Adverse events detected by clinical surveillance on an obstetric service. Obstet Gynecol. 2006; 108:1073-83.

24. Thomas EJ, Lipsitz SR, Studdert DM, Brennan TA. The reliability of medical record review for estimating adverse event rates. Ann Intern Med. 2002; 136(11):812-6. 